msh-mss Mathématiques et sciences humaines

182 | Été 2008

Varia

\title{
Inégalité des chances et agressivité motrice
}

Unfair sporting games and motor aggressiveness

\section{Luc Collard}

\section{(2) OpenEdition}

\section{Journals}

Édition électronique

URL : http://journals.openedition.org/msh/10293

DOI : $10.4000 / \mathrm{msh} .10293$

ISSN : 1950-6821

\section{Éditeur}

Centre d'analyse et de mathématique sociales de l'EHESS

\section{Édition imprimée}

Date de publication : 30 juin 2008

Pagination : 33-46

ISSN : 0987-6936

\section{Référence électronique}

Luc Collard, «Inégalité des chances et agressivité motrice », Mathématiques et sciences humaines [En ligne], 182 | Été 2008, mis en ligne le 30 juin 2008, consulté le 23 juillet 2020. URL : http:// journals.openedition.org/msh/10293; DOI : https://doi.org/10.4000/msh.10293 


\title{
INÉGALITÉ DES CHANCES ET AGRESSIVITÉ MOTRICE
}

\author{
Luc COLLARD $^{1}$
}

\begin{abstract}
RÉSUMÉ - Il s'agit de vérifier sur le plan expérimental une hypothèse d'Elias [1986] selon laquelle, dans les jeux sportifs, l'inégalité des chances serait source de déchaînements agressifs. Pour cela, des adultes volontaires sont soumis en $6 \sqcup 6$ à un jeu sportif inéquitable dépourvu d'équilibre tactique. Le jeu débute par le rendu d'une décision prise secrètement par chaque équipe : le choix d'un ballon sur deux possibles. Le score potentiel des uns est conditionnel au choix de ballon des autres. La matrice des gains est telle qu'à chaque coup l'une des deux équipes ressort frustrée de son choix.

L'originalité de la recherche consiste alors à provoquer la résolution du jeu par l'accomplissement moteur. Partant d'un bout à l'autre d'un gymnase, les équipes s'élancent et se croisent. Elles cherchent à plaquer leur ballon dans une zone opposée, condition nécessaire à l'obtention du score annoncé. Chaque équipe a toute liberté d'empêcher l'autre de parvenir à ses fins...

Le jeu étant répété, les stratégies mixtes liminaires apparaissent plutôt rationnelles. Par contre, au moment des réalisations motrices, les équipes minimisent leurs gains en se faisant vengeance. Les joueurs
\end{abstract} se livrent à des actions d'autant plus agressives que le rapport du jeu leur est défavorable.

MOTS-CLÉS - Agressivité motrice, Jeu sportif, Stratégie mixte, Théorie des jeux expérimentale

SUMMARY - Unfair sporting games and motor aggressiveness

The goal of the present work was to experimentally verify Elias's hypothesis [1986], according to which unfair sporting situations lead to aggressive outbursts. To this end, adult volunteers were subjected to an inherently unfair 6-a-side game which lacked a balanced tactical basis. At the start of the game, a decision is taken separately by each team: the choice of one of two possible balls. One team's potential score depends on the choice of the ball made by the other team. The scoring matrix is designed in such a way that every time a point is scored, one of the two teams feels penalized by its opening choice.

The originality of the present work consisted in ending the game with a potentially aggressive motor action. Starting from opposite ends of a sports hall, the two teams have to touch their ball down in the opposing end zone in order to definitively acquire the previously scored points. Each team is free to prevent the other one from achieving its objective by any means it sees fit...

When the game is replayed, the initial "mixed" strategies appear to be quite rational. On the other hand, when the motor action has to be performed, the teams minimize their gains whilst taking their revenge on their opponents. The more distorted the score, the more aggressive the players. game

KEY-WORDS - Experimental game theory, Mixed strategy, Motor aggressiveness, Sporting

1 CRAPS [EA 2131], UFR STAPS de Caen, boulevard du Maréchal Juin, Campus II, 14032 Caen cedex, luc.collard@orange.fr 


\section{INTRODUCTION. POURQUOI SOUMETTRE DES JOUEURS À UN JEU SPORTIF INÉQUITABLE SANS ÉQUILIBRE TACTIQUE?}

Dans un ouvrage co-écrit avec Eric Dunning [1986], Norbert Elias avance une hypothèse qui semble à première vue banale. Le sport protégerait les participants de conduites désordonnées et violentes en leur garantissant dès le départ une parfaite égalité des chances. Le sociologue ne prétend pas cette condition suffisante. Il faudrait en effet ajouter des précisions sur les distances d'affrontement, l'espace d'action et l'espace-cible, les types d'interactions motrices privilégiées ; en somme : préciser les autres traits de logique interne exerçant une influence coercitive sur les conduites motrices des joueurs [Parlebas, 1999]. Mais l'originalité de l'hypothèse d'Elias est d'envisager l'équité du système de réussite comme un agent de pacification des mœurs.

Dès lors que les opposants se soumettent à des règles garantissant une équiprobabilité de réussite ou d'échec associée à des espérances de gains semblables, le perdant n'a plus à considérer l'échec comme une offense nécessitant vengeance et le vainqueur peut « jouir de la confirmation de sa propre valeur sans mauvaise conscience,..., dans la certitude que le combat a été loyal » écrit Elias [1986, p. 64]. Pierre Parlebas [2005] confirme que les compétitions sportives occidentales reposent aujourd'hui sur une structure de coalitions qui est connexe, stable, symétrique, exclusive et équilibrée. Il s'agit d'une « véritable épure » souligne l'auteur, dont nous pouvons avancer qu'elle participe à la censure des conduites désordonnées dirigées sur autrui.

Que se passerait-il alors dans un jeu sportif où l'égalité des chances serait purement et simplement remise en question ? Est-il possible d'imaginer un jeu injuste auquel des joueurs pourraient accepter de se livrer physiquement? Observerions-nous d'autant plus d'agressivité à l'encontre de joueurs profitant de gains immérités ? Impossible de répondre à ces interrogations sans recourir à l'observation de joueurs en chair et en os. L'article qui suit tente de relever ce défi en joignant à la Théorie des jeux expérimentale [Kagel, Roth, 1995] une contrainte supplémentaire : la prise en compte de l'action motrice. Pour cela, il nous faut partir d'une situation motrice peu commune et disons même peu naturelle - tant l'équité s'est imposée en sport comme une donnée fondamentale.

Nous retenons un modèle de jeu emprunté à Marc Barbut [1967] dans un article consacré aux « Jeux qui ne sont pas de pur hasard ». L'auteur propose plusieurs matrices de jeux opposant deux joueurs, dont l'une - dissymétrique - a la particularité d'entraîner les protagonistes dans un cercle vicieux impossible à solutionner sans recourir au calcul probabiliste (cf. [Barbut, 1967, p. 851]). Pour les besoins de la recherche, nous nous permettons de modifier quelque peu la donne. Mais nous conservons le principe d'une ambiguïté permanente des décisions liminaires, y compris, comme cela va être le cas, lorsque l'on joue un grand nombre de fois. Tout l'intérêt du jeu est qu'il est injuste (pour un coup du jeu comparable, le pas de la marque n'est pas le même selon les joueurs, un peu comme si au football le fait de marquer un but rapportait 1 à une équipe et 4 à l'autre) et sans équilibre de Nash en stratégie pure (un des deux joueurs ayant toujours intérêt à changer de tactique si l'autre maintient la sienne). Cette double contrainte nous semble intéressante pour que vive le jeu. En effet, un jeu inéquitable mais équilibré sur le plan tactique (chaque joueur sachant exactement ce qu'il faut faire pour faire ce qu'il a de mieux à faire) conduirait rapidement les joueurs à s'en désintéresser. À quoi bon jouer à un jeu dont l'issue inique est courue d'avance ? Par contre, l'adjonction du non-équilibre tactique plonge les protagonistes dans la spirale stimulante d'un cercle vicieux où les joueurs lésés pensent toujours qu'ils auraient pu faire mieux. Voilà un contexte favorable au maintien d'intérêt et à d'éventuels règlements de comptes. 
La mise en jeu corporelle de ce modèle nous invite à distinguer deux formes de décision :

- Une décision de type cognitif faisant appel aux «deux sources de probabilités » [Condorcet, 1785] : l'une renferme les probabilités tirées de l'estimation objective des chances, compte tenu de la conduite rationnelle anticipée de l'autre et selon les gains possibles du jeu. L'autre source de probabilité est fondée sur l'expérience du passé, « les motifs de croire », attribuant une utilité subjective au risque encouru.

- Et une décision motrice présentant l'originalité de prendre corps dans un comportement moteur, au cours même du flux de l'action. « Manifestant dans son accomplissement un choix lié à une incertitude », elle participe « sur le champ à la résolution de la tâche motrice » (cf. [Parlebas, 1999, p. 90]).

Répondre à notre problématique revient à croiser ces deux formes de décision aux comportements logiques rendus par le calcul. Comment les joueurs devraient-ils jouer et comment jouent-ils réellement (notamment au moment de l'action motrice) ?

\title{
2. DÉROULEMENT DU JEU, CADRE DE L'EXPÉRIENCE
}

À chaque coup du jeu, deux équipes de six joueurs se voient attribuer un prix à atteindre en fonction du ballon qu'elles choisissent. Si elles choisissent toutes deux un ballon de handball (HB), il vient un score potentiel de 2 pour l'équipe A et 3 pour l'équipe B ; un ballon de HB pour A et de GR (Gymnastique Rythmique) pour B donnent respectivement $\{3,1\}$; l'inverse (GR pour A, HB pour B) donne $\{4,2\}$; enfin, deux ballons de GR choisis donnent $\{1,4\}$ (cf. Figure 1). Le choix du ballon d'une équipe n'est connu de l'autre que quelques instants avant la mise en jeu pratique de chaque partie. Pour pouvoir remporter le prix annoncé, une équipe doit traverser le terrain $(40 \mathrm{~m}$ de long sur $20 \mathrm{~m}$ de large) et aller poser sa balle dans le camp adverse (zone des $6 \mathrm{~m}$ du Handball). L'autre équipe fait de même en sens inverse. Les équipes se croisent. Elles peuvent ainsi se gêner et même empêcher les autres de marquer en leur subtilisant le ballon.

\begin{tabular}{lccc} 
& & \multicolumn{2}{c}{ Équipe B } \\
& & ballon de HB & ballon de GR \\
\cline { 3 - 4 } Équipe A & ballon de HB & $\{2,3\}$ & $\{3,1\}$ \\
& ballon de GR & $\{4,2\}$ & $\{1,4\}$ \\
\cline { 3 - 4 } & &
\end{tabular}

FIGURE 1. Matrice des gains en fonction du ballon choisi

\begin{abstract}
Dans chacune des 4 cases, le premier chiffre entre parenthèses correspond au gain de l'équipe A, et le second chiffre au gain de l'équipe B. Pour maximiser leurs gains, on voit très vite que l'une des deux équipes a toujours intérêt à changer de tactique (choix d'un ballon différent) si l'autre maintient la sienne.
\end{abstract}

C'est ici la différence fondamentale avec les modèles classiques de la Théorie des jeux dans lesquels, une fois la décision prise, les joueurs n'ont qu'à prendre leur mal en patience et attendre le paiement de la partie. Par contre, pour nos sportifs, une fois les tactiques des deux équipes annoncées, c'est ici que le jeu commence et qu'il va falloir faire preuve d'adresse...

Cela dit, l'opposition n'est pas imposée. Les deux équipes peuvent très bien coopérer. Cela se traduit sur le champ de jeu par un laissez-passer du porteur de balle adverse. Le but du jeu est clairement annoncé. Il ne s'agit pas de terrasser l'équipe adverse, mais d'empocher le plus de points possibles afin de s'approcher du score 
théorique maximum (score culminant de la matrice : 4 points, multiplié par le nombre de parties). Car l'expérience porte sur 4 semaines (2 équipes par semaine) et toutes les équipes concernées ne se rencontreront pas. Le classement final désignera victorieuse l'équipe parmi les 8 participantes dont le score sera le plus élevé. Les choses sont dites. Attention donc, sur le champ de jeu, aux conduites rancunières qui pourraient se solder par des neutralisations de balles et la perte des points qui leur sont associés.

Tout est permis dans les relations interindividuelles et aucune limite spatiale autre que les murs du gymnase ne vient les contenir. Les règles habituelles visant à codifier les conduites étant écartées, on peut alors penser que les décisions motrices se feront essentiellement sous l'emprise de ce contraignant système des scores. L'expérience porte sur 4 rencontres de 2 équipes de 6 sportifs comparables (20-23 ans), soit un total de 48 joueurs. Chaque équipe joue 20 parties. L'action motrice de chaque partie dure en moyenne 1 minute; le temps de traverser «à toute pompe » un terrain de $40 \mathrm{~m}$ potentiellement semé d'embûches... Les 80 parties de l'expérience sont filmées par 2 caméras en position surélevée qui suivent chacune le déplacement d'une équipe. Tous les joueurs sont des adultes volontaires étudiant dans une même faculté française des sciences du sport.

Répétons-nous. Chaque partie se décompose en 2 temps successifs :

- d'abord, rassemblée dans leur camp respectif, chaque équipe choisit en secret une des deux balles conformément à la matrice des gains affichée (cf. Figure 1) ;

- ayant pris connaissance du choix de ballon adverse, de la combinaison tactique et des scores correspondants, les équipes s'élancent de part et d'autre du gymnase, décidées à plaquer leur balle dans le camp adverse, seule façon d'empocher les gains annoncés.

Un tel jeu peut s'analyser « à rebours » (en commençant à raisonner à partir de la fin, au moment de l'action motrice) car les comportements sur le terrain peuvent être différents suivant le choix de ballons initiaux ; la décision rationnelle du choix du ballon devant alors reposer sur l'anticipation des comportements sur le terrain.

\section{ESTIMATION DES COMPORTEMENTS LOGIQUES AVANT DE JOUER}

\subsection{RÉSOLUTION PAR RÉCURRENCE À REBOURS}

Commençons donc par estimer les décisions motrices rationnelles au moment de traverser le gymnase. Pour cela, représentons d'abord le jeu sous forme d'un arbre (cf. Figure 2). Dans cette configuration - dite sous forme extensive - le sommet de l'arbre représente le coup initial, autrement dit le rendu liminaire simultané des choix de ballon par les équipes $\mathrm{A}$ et $\mathrm{B}$. Les 4 branches issues de ce sommet aboutissent à 4 nouds correspondant aux 4 combinaisons de tactiques possibles : $\{\mathrm{HB}, \mathrm{HB}\},\{\mathrm{HB}, \mathrm{GR}\},\{\mathrm{GR}, \mathrm{HB}\}$ et $\{\mathrm{GR}, \mathrm{GR}\}$. De ces 4 combinaisons partent 4 nouvelles branches représentant les gains obtenus selon que les équipes laissent jouer (Co pour «Conciliant») ou s'opposent ( $A g$ pour «Agressif») sur le champ de jeu. Si A et B neutralisent de concert le ballon adverse en jouant agressif $\{A g, A g\}$, ils obtiennent leur plus mauvais score : $\{0,0\}$. Si, par contre, ils jouent la pure coopération $\{C o, C o\}$, ils obtiennent tous deux leur meilleur score : $\{2,3\},\{1,4\},\{3,1\}$ ou $\{4,2\}$. Chaque équipe peut bien sûr augmenter son avance sur l'autre :\{2,0\}\{1,0\}, $\{3,0\}$ ou $\{4,0\}$ en faveur de $A$, par exemple (le premier chiffre dans la parenthèse est toujours le score de A). Il faut pour cela que l'équipe B se fasse subtiliser sa balle tout en laissant passer le ballon de $\mathrm{A}\{A g, C o\}$. Adoptée de façon intentionnelle, une telle conduite ne 
serait guère logique pour B. À quoi bon être conciliant avec un agresseur qui limite ipso facto vos gains ? Cette situation est même très peu probable en pratique.

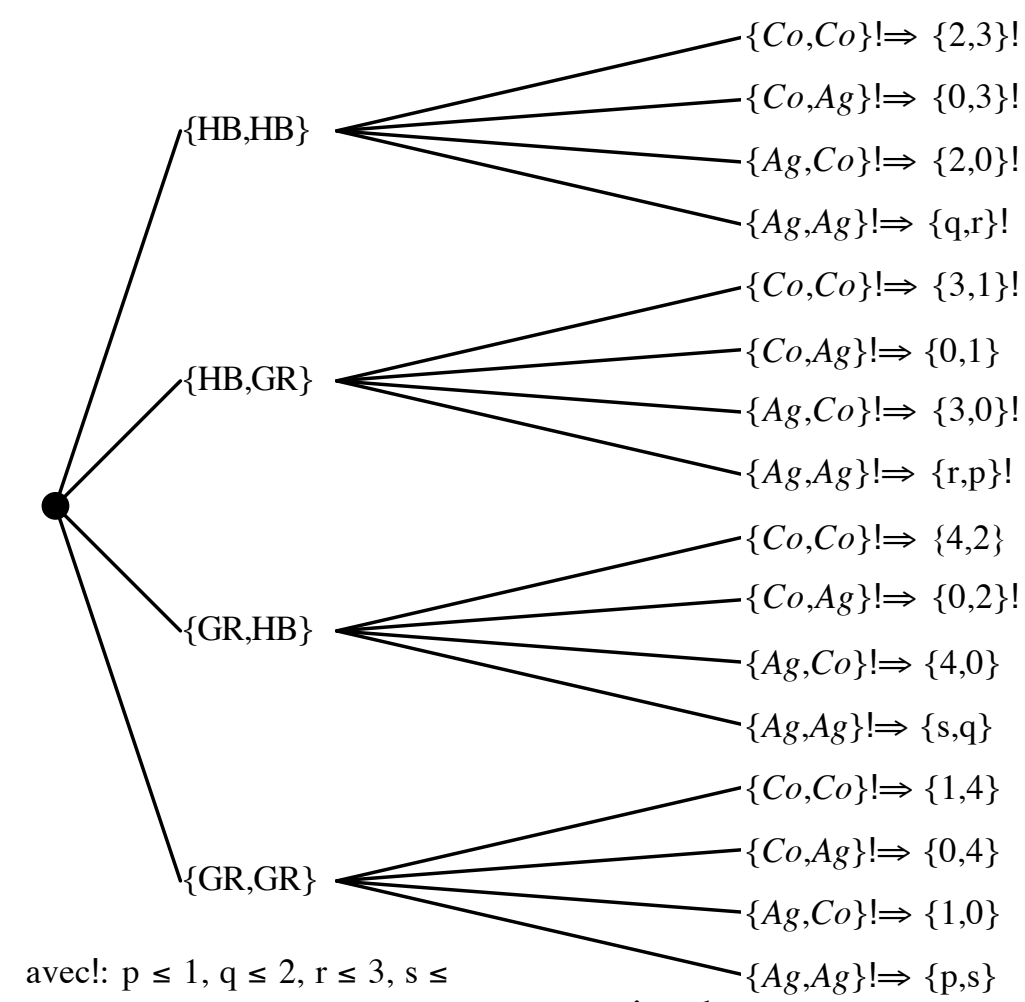

FIGURE 2. Présentation sous torme extensive des 2 moments-clets du jeu

Partant du sommet de l'arbre, les 4 branches aboutissent aux 4 combinaisons de choix de ballon. De ces 4 combinaisons partent 4 branches correspondant aux conduites de terrain potentiellement endossées : les 2 équipes pouvant jouer Agressif $(\mathrm{Ag})$ ou Conciliant $(\mathrm{Co})$. La récurrence à rebours révèle une tactique dominante au moment de l'action motrice : coopérer. En effet, sur le terrain, les combinaisons $\{C o, A g\}$ et $\{A g, C o\}$ ne sont pas viables (un des 2 protagonistes ayant alors toujours intérêt à faire volte face au cours même de la situation motrice) et $\{C o, C o\}$ domine $\{A g, A g\}$ qui peut aboutir au pire à $\{0,0\}$ (en cas de neutralisation réciproque des ballons avant la cible).

Le jeu étant à information complète au moment de l'action motrice, une équipe s'apprêtant à être conciliante $(\mathrm{Co})$ ne restera pas insensible à l'agressivité adverse $(\mathrm{Ag})$ et profitera de la minute que dure une partie pour tourner casaque et jouer agressif à son tour $\{A g, A g\}$.

De plus, le jeu étant répété (20 fois), on peut penser que la trahison d'un «pacte coopératif » se paiera cher les coups suivants. Bien qu'a priori gentilles, les conduites «donnant-donnant » (« La $1^{\text {ère }}$ partie je joue Co et, les coups suivants je joue comme mon adversaire a joué au coup précédent ») et « graduelles » (« je coopère mais lorsque mon adversaire me trahit, je réplique par $\mathrm{N}$ coups agressifs, où $\mathrm{N}$ est le nombre de trahisons passées de mon adversaire...») dominent les conduites aléatoires ou lunatiques dans ce type de situation [Delahaye, 1998]. Ainsi, on ne devrait pas observer de combinaisons $\{A g, C o\}$ ou $\{C o, A g\}$.

Par contre, il se peut que deux équipes s'opposant simultanément, l'une fasse montre de plus d'habileté à « passer sans laisser passer » que l'autre. C'est la raison pour laquelle nous donnons des scores avec $\mathrm{p} \square 1, \mathrm{q} \square 2, \mathrm{r} \square 3$, s $\square 4$ et non pas $\{0,0\}$ lorsque 
les protagonistes jouent: $\{A g, A g\}$ (cf. Figure 2). Les chances de marquer sont alors tributaires de la dextérité motrice des participants (esquive, contre, passage en force, etc.). Mais le risque est grand de perdre bêtement des points par co-neutralisation. Points qui auraient pu automatiquement revenir en cas de coopération. Sur le terrain, ce qui compte c'est d'atteindre le chiffre le plus élevé possible au bout de 20 parties, quoi que fasse l'autre. C'est bien dans cet esprit que le jeu a été présenté.

Que l'équipe opposée débute le jeu avec un capital plus élevé de points est un manque de chance que la punition ne contrecarrera pas. Les joueurs se condamnent en s'affrontant manu militari. L'équipe partant avec une espérance de gain moindre doit ravaler sa rancune et espérer une meilleure donne au coup suivant...

D'un point de vue rationnel, on devrait donc voir des équipes qui se croisent en s'évitant courtoisement. Nous en convenons aisément : conduire un ballon sur $40 \mathrm{~m}$ sans rencontrer d'obstacle n'a rien de palpitant sur le plan de l'accomplissement moteur. Cela dit, les nerfs des joueurs sont mis à rude épreuve. Il faut un degré élevé d'autocontrainte pour laisser complaisamment son adversaire - profitant de gains supérieurs - porter son ballon dans l'espace cible sans coup férir. C'est exactement le contexte de tensions que nous recherchons. Si nous avions soumis les joueurs à un duel sportif - où tout ce que l'un gagne, l'autre le perd - nous aurions artificiellement déclenché de l'adversité et des résistances, que le jeu soit équitable ou pas. Ici, tout écart aux conduites de coopération sera symptomatique d'actions passionnées visant à compenser un déloyal système des scores.

Balle en main, les équipes jouent un jeu dépourvu de toute ambiguïté. Quelle que soit la donne initiale et afin de maximiser leurs gains, les joueurs gagnent à coopérer avec l'équipe d'en face $\{C o, C o\}$. La récurrence à rebours ne permet finalement pas d'éclairer les choix de ballon initiaux. L'arbre se réduit après «élagage » au jeu inéquitable de départ, toujours dépourvu d'équilibre tactique (cf. Figure 3).

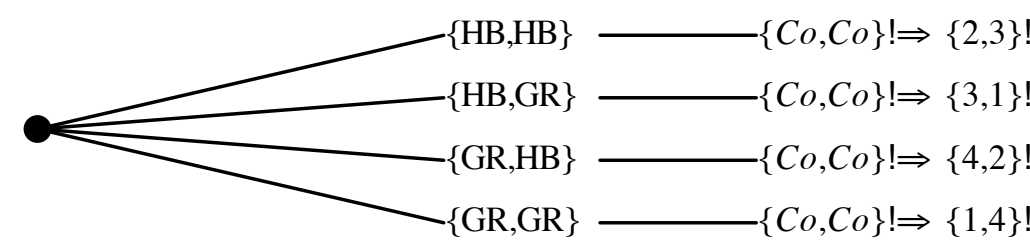

FIGURE 3. Jeu obtenu après récurrence à rebours

Même si la conduite de terrain est sans ambiguïté $\{C o, C o\}$, cela n'éclaire en rien le choix initial de ballon à adopter. Une des 2 équipes ayant toujours à regretter son choix. 


\subsection{DÉCISIONS COGNITIVES RENDUES PAR LE CALCUL}

Estimant que la tactique la plus prudente de l'équipe $\mathrm{B}$ - son moindre mal - est de jouer avec le ballon HB (au pire elle aura 2), l'équipe A peut ruser en choisissant le ballon GR et espérer ainsi empocher 4. Mais l'équipe B peut s'en douter et jouer aussi GR. Elle peut alors gagner 4 et l'équipe A ne joue plus que pour 1 . Anticipant cette anticipation, A peut espérer 3 en choisissant le ballon HB si B joue GR, mais seulement 2, et B : 3, si chacun choisit le ballon HB... Et ainsi de suite... Les protagonistes sont pris dans un cercle vicieux. Si la situation n'admet pas de solution en stratégie pure, sa résolution est possible en usant de probabilités et d'espérances mathématiques. On parle alors de résolution en stratégie mixte.

On appelle $h b$ et $g r$ les probabilités pour l'équipe A de jouer avec les ballons de HB et de GR. Soit M la valeur du moindre mal en stratégie mixte.

Si $\mathrm{B}$ joue ballon $\mathrm{HB}$, A recevra $(2 h b+4 g r)$, c'est-à-dire son espérance mathématique. Si B joue ballon GR, A recevra $(3 h b+1 g r)$, soit encore son espérance mathématique. Marc Barbut [1967] montre que l'on doit résoudre :

$$
\begin{aligned}
& 2 h b+4 g r=\mathrm{M}, \\
& 3 h b+1 g r=\mathrm{M}, \\
& h b+g r=1 .
\end{aligned}
$$

Il vient $h b=$, c'est-à-dire que si A joue 3 fois sur 4 avec le ballon HB (et donc 1 fois sur 4 avec le ballon GR), il améliore son gain et réduit sa perte. Du fait de la symétrie des scores, que A joue HB ou GR, l'équipe B optimise également ses gains potentiels en jouant avec une probabilité de _ avec le ballon HB et avec une probabilité de _ avec le ballon GR. L'espérance de gain est alors de $M=+5 / 2$ pour les deux camps. C'est le mieux de ce qu'ils peuvent faire pour limiter le pire qui pourrait leur arriver : c'est le maximum de leur minimum, leur maximin.

Hélas, cette situation n'est pas équilibrée. Si B joue son moindre mal (trois fois sur quatre avec le ballon HB), A améliorera son gain en choisissant par exemple $h b<_{-}$.

"L'essence d'une situation équilibrée au sens de Cournot, écrit Barbut [1967, p. 855], c'est d'être pour chacun des adversaires, ce qu'il peut faire de mieux en réaction à l'action de l'autre ». Si nous raisonnons par rapport à l'espérance de A, nous serons en situation équilibrée si la stratégie de $\mathrm{B}$ est telle que, quoi que fasse $\mathrm{A}$, il ne puisse améliorer son espérance. Autrement dit, l'équilibre (E) s'obtient lorsque B joue avec des probabilités $h b$ et $g r$ telles que, quoi qu'il fasse, A ne puisse améliorer son sort. Le calcul fournit dans ce cas :

$2 h b+3(1-h b)=\mathrm{E}$, si A joue avec le ballon HB et,

$4 h b+1-h b=\mathrm{E}$, si A joue avec le ballon GR.

Il vient $h b=\_, g r=\ldots$ et $\mathrm{E}=+5 / 2$, pour A comme pour B. Si les concurrents jouent une fois sur deux avec le ballon HB ou le ballon GR, on remarque qu'ils ont une espérance équivalente à ce que leur donne le moindre mal $(\mathrm{M}=+5 / 2)$ mais avec la garantie qu'aucun des deux n'a intérêt à changer de tactique si l'autre maintient la sienne. Hélas, bien qu'en situation équilibrée, le sort de chaque équipe dépend alors terriblement de l'autre. La tentation peut être grande de chercher à dégrader délibérément le sort de l'autre. En choisissant de jouer le ballon $\mathrm{HB}$ avec une probabilité $h b>_{\text {, }}$, imaginons l'extrême : $h b$ $=1$, A a le pouvoir de réduire l'espérance de $\mathrm{B}-$ qui continue loyalement à jouer l'équilibre, à 2 (au lieu des $+5 / 2$ ). Alors qu'en situation de moindre mal, l'espérance est indépendante de ce que font les adversaires. Mais la tentation est ici grande de chercher à 
changer de tactique pour améliorer son sort et finalement risquer d'obtenir moins. Comment réagiront nos joueurs ? Vont-ils préférer l'assurance du moindre mal en évitant les coups bas de l'adversaire ou vont-ils choisir de concert l'équilibre ?

\section{RÉSULTATS. CONDUITES OBSERVÉES DURANT LE JEU}

\subsection{DÉCISIONS COGNITIVES LIMINAIRES : DES CHOIX PLUTÔT ÉQUILIBRÉS ET PRUDENTS}

Pour les 8 équipes d'étudiants sportifs volontaires observées et sur les 20 parties à chaque fois réitérées, le ballon HB a été choisi majoritairement : 101 sur 160 choix distincts (80 parties, 2 équipes), soit environ dans $5 / 8^{\text {ème }}$ des cas. Une telle stratégie mixte liminaire est intermédiaire entre leur moindre mal où $h b={ }_{-}$et l'équilibre où $h b=\ldots$. Les joueurs semblent avoir hésité entre la protection instable et la stabilité non protégée.

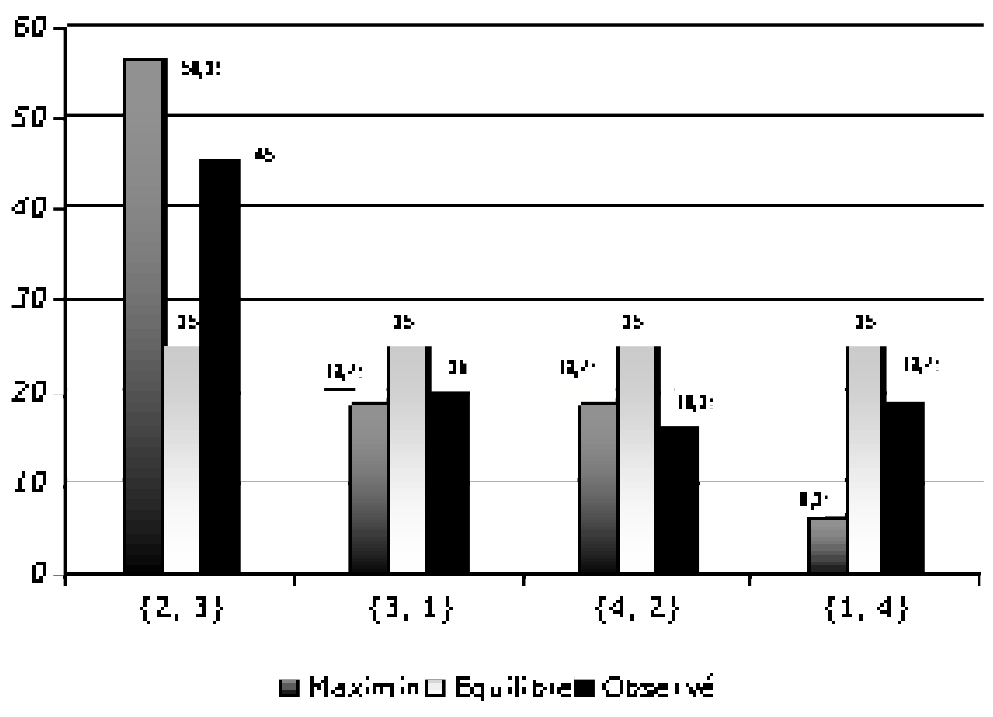

FIGURE 4. Maximin et équilibre rendus par le calcul en stratégies mixtes et décisions réelles prises par les sportifs sur 80 parties (résultats en \%)

On note que les choix liminaires des sportifs (45\% en $\{2,3\}, 20 \%$ en $\{3,1\}, 16 \%$ en $\{4,2\}$ et $19 \%$ en $\{1,4\})$ sont globalement intermédiaires entre, ce que donne l'équilibre et ce que donne le moindre mal.

La combinaison la plus prudente $\{2,3\}$, représentée par le choix simultané d'un ballon HB, a été retenue à 36 reprises (45\% des cas). Si chaque équipe jouait son maximin, il y aurait $\left({ }_{-}{ }_{-}\right)_{-} 80=45$ parties sur 80 (soit 56\%) de séquences de jeu avec les 2 ballons de handball. Si par contre, les 2 équipes jouaient l'équilibre, il y aurait (_ _ _ _ $80=20$ parties sur 80 (soit $25 \%$ ) de séquences de jeu avec les 2 ballons de handball. Avec $45 \%$ de combinaisons $\{2,3\}$ on est presque à la moyenne du moindre mal et de l'équilibre. Quant aux 3 autres combinaisons $\{3,1\},\{4,2\},\{1,4\}$, elles se partagent plutôt équitablement les $55 \%$ restants (cf. Figure 4). Leurs fréquences d'apparition sont également proches des résultats théoriques faisant la moyenne de l'équilibre et du maximin (moindre mal). 


\subsection{DÉCISIONS MOTRICES : DES CONDUITES VENGERESSES IMPRÉGNÉES D’AFFECTIVITÉ}

Sur le champ de jeu par contre, les résultats se gâtent. Ainsi que le montre la Figure 5, dans les 4 cas de figure $(\{2,3\},\{1,4\},\{3,1\},\{4,2\})$, les scores obtenus sont systématiquement inférieurs aux scores théoriques si A et B jouaient la stricte coopération. Ceci est symptomatique des affrontements ludiques obstinés qui ont empêché les ballons - dans 7 à $44 \%$ des cas - d'atteindre leur cible.

\begin{tabular}{|c|c|c|c|c|c|}
\hline $\begin{array}{c}\text { N: Fréquence } \\
\text { des } \\
\text { combinaisons } \\
\text { tactiques sur } 80 \\
\text { parties }\end{array}$ & $\begin{array}{c}\text { Combinaisons } \\
\text { tactiques }\end{array}$ & $\begin{array}{c}\text { Score théorique } \\
\text { si coopération/N }\end{array}$ & Score réel/N & $\begin{array}{c}\text { Manque } \\
\text { à gagner }\end{array}$ & $\begin{array}{c}\text { Taux } \\
\text { de } \\
\text { perte }\end{array}$ \\
\hline \multirow{2}{*}{36} & $\{2$, & $36 \_2=72$ & 52 & -20 & $28 \%$ \\
\cline { 3 - 6 } & $3\}$ & $36 \_3=108$ & 78 & -30 & $28 \%$ \\
\hline \multirow{2}{*}{15} & $\{1$, & $15 \_1=15$ & 14 & -1 & $7 \%$ \\
\cline { 3 - 6 } & $4\}$ & $15 \_4=60$ & 44 & -16 & $27 \%$ \\
\hline \multirow{2}{*}{16} & $\{3$, & $16 \_3=48$ & 27 & -21 & $44 \%$ \\
\cline { 3 - 6 } & $1\}$ & $16 \_1=16$ & 10 & -6 & $37 \%$ \\
\hline \multirow{2}{*}{13} & $\{4$, & $13 \_4=52$ & 32 & -20 & $38 \%$ \\
\cline { 3 - 6 } & $2\}$ & $13 \_2=26$ & 16 & -10 & $38 \%$ \\
\hline
\end{tabular}

FIGURE 5 : Croisement des scores optimaux si coopération, et réels sur les 80 parties observées

Quelles que soient les combinaisons liminaires de tactiques, les équipes obtiennent systématiquement sur le champ de jeu des scores inférieurs à ce qu'elles auraient pu obtenir, signe qu'elles n'ont pas joué la pure coopération.

6a - combinaisons

$\{2,3\}$

Conciliant

Agressif

Conciliant Agressif

\begin{tabular}{|cc|}
\hline-1 & -3 \\
2 & $\mathbf{0}$ \\
\hline
\end{tabular}

$6 \mathrm{~b}-$ combinaisons

$\{1,4\}$

Conciliant

Agressif
$6 c-$ combinaisons

$$
\{3,1\}
$$

Conciliant

Agressif

\begin{tabular}{|cc|} 
Conciliant & Agressif \\
\hline 2 & -1 \\
3 & $\mathbf{0}$ \\
\hline
\end{tabular}

$6 c-$ combinaisons

Conciliant

Agressif

\begin{tabular}{|cc|}
\hline \multicolumn{1}{c}{ Conciliant } & Agressif \\
\hline 2 & -2 \\
4 & $\mathbf{0}$ \\
\hline
\end{tabular}

FIGURE 6. Jeu auquel ont effectivement joué les étudiants sportifs.

Les matrices présentent les scores de l'équipe-ligne (équipe A).

La logique est devenue celle d'un duel

Dans la logique de duel adoptée et dans les 4 situations (a, b, c, d), chaque équipe gagne à présent à s'opposer à l'autre (maximin $=$ équilibre $=\{A g, A g\})$. Hélas cette situation est une réinterprétation erronée du jeu. Rappelons que le but du jeu était d'obtenir le meilleur score sur 20 parties, non d'empêcher l'autre équipe de marquer, au risque de ne pouvoir le faire soi-même $\{0,0\}$.

Les joueurs ont très clairement transgressé l'objectif annoncé. La tentation était grande en effet, face à un score défavorable (c'est toujours le cas à chaque coup pour une des deux équipes), de se faire vengeance et d'empêcher l'équipe adverse de profiter de son 
avantage. Dans les faits, tout s'est passé comme si les joueurs se soumettaient à la logique d'un duel dissymétrique (cf. Figure 6).

\subsubsection{Stratégie de terrain après avoir pris connaissance du choix de ballon adverse}

L'élément le plus visible de la recherche d'opposition systématique a été l'adaptation permanente du nombre de joueurs en attaque et en défense. Du point de vue rationnel de la Théorie des jeux, tous les joueurs auraient dû être en attaque, concentrés sur le devenir de leur propre ballon et insensibles à celui de l'équipe d'en face. Or, dès l'annonce du score convoité et avant le coup d'envoi, les équipes de 6 joueurs se concertaient rapidement dans leur camp. Combien d'attaquants seraient concernés pour conduire la balle dans la zone adverse ? Combien de défenseurs auraient pour mission d'empêcher le ballon adverse de se poser dans leur propre camp ? Tout dépend, avons-nous constaté, du produit du jeu, c'est-à-dire du gain ou de la perte calculés en soustrayant ce que l'on peut gagner de ce que peut gagner l'adversaire. Par exemple, si les tactiques liminaires imposent un rapport de jeu défavorable pour l'équipe $\mathrm{A}$, disons de 1 pour 4 (cas où les deux sous-groupes ont retenu le ballon GR), le produit du jeu est de -3 pour A $(1-4)$ et +3 pour B $(-1+4)$ si chaque équipe parvient à marquer.

\begin{tabular}{|c|c|c|c|c|c|c|c|}
\hline $\begin{array}{c}\text { Rapport } \\
\text { attaquant-défenseur } \rightarrow \\
\text { Produit du jeu } \downarrow \\
\end{array}$ & 6-0 & $5-1$ & $4-2$ & $3-3$ & $2-4$ & $1-5$ & $0-6$ \\
\hline+3 & & 8 & 6 & 1 & & & \\
\hline+2 & & 7 & 12 & 10 & & & \\
\hline+1 & & & 9 & 24 & 3 & & \\
\hline-1 & & & 8 & 16 & 11 & 1 & \\
\hline-2 & & & & 5 & 9 & 15 & \\
\hline-3 & & & & & 2 & 13 & \\
\hline
\end{tabular}

FIGURE 7. Produits du jeu et répartition du nombre d'attaquants et de défenseurs au début de chaque coup

(80 parties_ 2 équipes $=160$ distributions attaque-défense)

En ligne, les chiffres annoncent d'abord le nombre d'attaquants, puis, suivi d'un tiret, le nombre de défenseurs. 3-3 signifie qu'il y a autant des deux. En colonne, on a le « produit » du jeu, c'est-à-dire le gain ou la perte de chaque combinaison tactique. On observe logiquement une propension marquée en faveur de l'attaque (5-1, 4-2) lorsque le rapport du jeu est favorable, et un bouclier défensif $(2-4,1-5)$ en cas contraire.

L'analyse des films nous conduit à penser qu'en situation $\{1,4\}$ l'équipe $\mathrm{A}$ a estimé qu'il valait mieux «mettre le paquet » en défense et limiter le nombre d'attaquants. En effet, si tous les joueurs de l'équipe $\mathrm{A}$ se mettent en défense - renonçant ainsi à conduire leur propre ballon dans la zone cible, et parviennent à intercepter la balle de $\mathrm{B}$, le produit réel du jeu sera égal à zéro, ce qui est mieux que -3 (cf. Figure 6b). Par contre, l'équipe $\mathrm{B}$ a tout intérêt à mettre plus d'attaquants que de défenseurs. Si elle réussit dans la réalisation de sa tactique elle empochera +3 si $\mathrm{A}$ réussit de son côté à marquer, et +4 si A échoue. On peut légitimement penser que dans cet esprit de jeu strictement compétitif, cette séquence de jeu opposera 6 défenseurs A à 6 attaquants B. L'expérience montre une position moins extrême des joueurs, mais tout de même dans ce sens (cf. Figure 7). 
Les sportifs observés ont joué en estimant que, pour gagner, il fallait s'opposer à l'autre. Non seulement cela n'était pas nécessaire, mais cela les a conduits à perdre plus. La logique interne du jeu invitait les protagonistes à se focaliser sur leur propre gain, pas sur le produit du jeu (leur propre gain moins celui de l'autre). En effet, sur le vif, les interactions motrices d'opposition se soldent inéluctablement par des neutralisations de balle, sorte de « co-destruction ».

En en discutant avec les joueurs, nous avons compris qu'ils avaient bien perçu le danger de livrer bataille. Deux arguments ont été avancés de façon redondante :

- D'abord, sur le plan de l'action motrice, se contenter d'un laisser-passer conciliant aurait donné un jeu sans entrain et particulièrement frustrant ; une des deux équipes ayant toujours à l'esprit qu'elle va empocher moins que l'autre.

- Ensuite, les équipes se sont senties capables de conquérir le score convoité tout en empêchant l'autre de parvenir à ses fins ; ce sentiment de supériorité bilatérale sur le plan de l'action motrice (qui a permis tout de même à une majorité d'assauts d'aboutir (cf. Figure 5)) a occasionné la perte de nombreux points (de 7 à $44 \%$ du maximum possible). Des dominants dominés par leur domination, en quelque sorte.

\subsubsection{Exécution sur le terrain d'actions motrices agressives}

Aucune règle ne venant limiter les interactions motrices, nous avons observé sur le champ de jeu des comportements agressifs d'ampleur variable. On peut distinguer 3 classes d'agressivité de pertinence motrice :

- D'abord, les manifestations visant à gêner l'adversaire, sans pénétrer dans son espace intime en mode proche (de 0 à $15 \mathrm{~cm}$, pour reprendre la typologie de Hall [1966]). Il s'agit des actes nécessitant le plus d'autocontrôle : harceler, faire obstruction, marquer de près, etc. Tout cela sans contact. C'est l'agressivité physique hyper-ritualisée, telle qu'elle est privilégiée dans nos sports collectifs. Nous isolons cette première catégorie dans la classe : " gênes ».

- Ensuite, on peut repérer une série de comportements agressifs visant à entrer en contact momentanément avec l'adversaire - bousculer, percuter, écarter, pousser, etc. Comme la première catégorie (et comme la troisième) elle peut être orientée aussi bien vers les porteurs de balle que vers les autres joueurs de champ. Il s'agit là d'un degré supérieur d'agressivité ne relevant plus seulement de l'intimidation mais usant de la force. Les joueurs touchés peuvent tomber, trébucher. Ce type d'agression est mis sous surveillance dans nos sports collectifs ; il arrive même qu'il soit légalement interdit comme en basket-ball et bien sûr en volley-ball. Nous le classons dans la catégorie : « contacts ».

- Enfin, il arrive que les joueurs fassent plus que percuter leurs assaillants. Ils peuvent s'agripper à eux, les plaquer au sol, les immobiliser physiquement, seul ou à plusieurs. Ce n'est plus une intrusion momentanée dans l'espace intime, c'est une mise sous tutelle de l'autre. Dans le règne animal, c'est par ce type d'actes que se forgent essentiellement les relations sociales de dominants et de dominés [Lorenz, 1969]. Le sport n'ignore pas complètement ce type d'agressivité mais s'arrange pour ne pas trop le faire durer : le rugbyman doit lâcher son ballon dès qu'il est plaqué, le judoka et le lutteur sont rapidement déclarés perdants dès qu'ils sont immobilisés. Nous désignons : «préhensions » de tels agissements. 


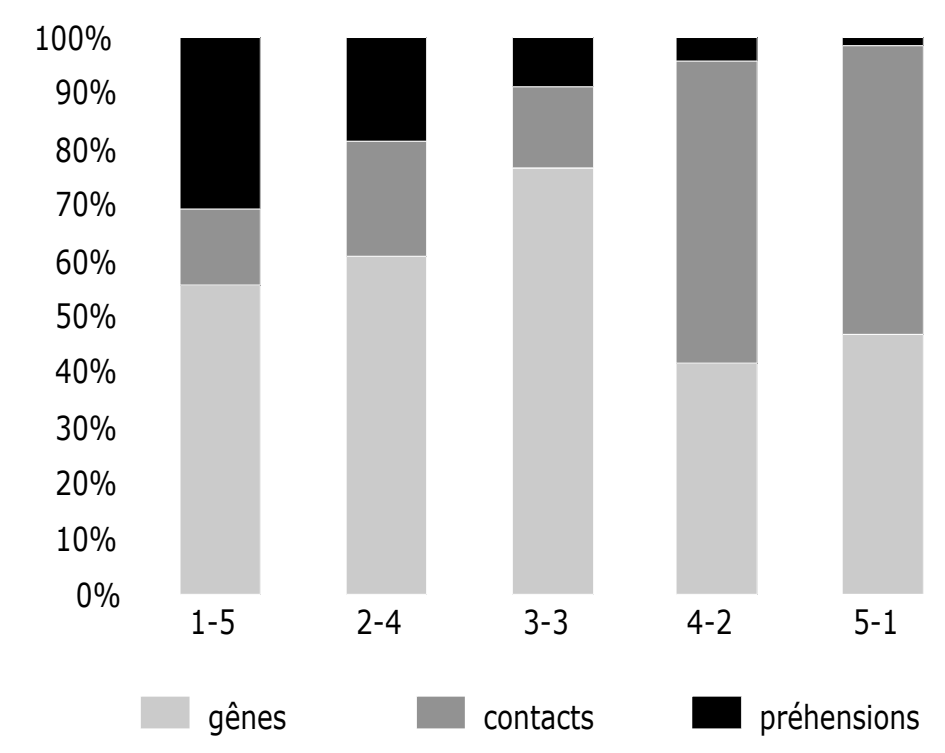

FIGURE 8. Comparaison, en pourcentage, des types d'agression en fonction du rapport attaquant-défenseur utilisé

On perçoit une présence forte de préhensions (en noir) lorsque l'on joue à 5 ou 4 en défense et 1 ou 2 en attaque : (1-5) et (2-4). Cette tendance disparaît lorsque le rapport entre attaquants et défenseurs s'inverse (5-1) et (4-2) : c'est le taux de contacts qui croît de façon forte. La disposition en (3-3) (autant de défenseurs que d'attaquants) valorise le mode agressif le plus édulcoré (les gênes). Les résultats sont significativement différents à $\mathrm{p}<0.01\left(\mathrm{KHI}^{2}\right)$.

La façon dont les joueurs se répartissent en attaque et en défense n'est pas étrangère à la nature de leurs agressivités (cf. Figure 8). En valorisant l'attaque (5-1) ou (4-2), un comportement agressif conduit plus facilement à un passage en force, type « contacts », qu'à la recherche d'immobilisation des adversaires, type «préhensions ». De fait, lorsque le produit du jeu est positif, l'attaque qui en découle tend à augmenter le taux de contacts par rapport aux autres types d'agression. À l'inverse, un produit de jeu négatif, générateur de boucliers défensifs, favorise l'émergence de "préhensions ». Nous comptabilisons près des deux tiers des actes de préhension dans deux des cinq dispositions valorisant la défense plutôt que l'attaque (2-4) et (1-5). Si, comme nous le suggérons, les actes de préhension constituent la forme la plus barbare d'agressivité corporelle, c'est plus dans la peur de perdre que dans la volonté de gagner qu'elle est prédisposée à s'exprimer.

En moyenne, les conduites agressives les plus pacifiées (gênes) se rencontrent dans $60 \%$ des cas (614 des 1025 actes agressifs comptabilisés sur 80 parties). Elles sont tributaires du rapport attaquant-défenseur ; mais lui-même est dicté par le produit du jeu (rappel de la Figure 7). Plus l'espérance de gain est réduite, plus il y aura de boucliers défensifs annonciateurs de préhensions. Ainsi, on recense deux fois plus de préhensions avec la combinaison de ballons menant à $\{1,4\}$ qu'en moyenne (20\% au lieu de 10). Par contre, lorsque les gains potentiels se rapprochent de l'équité $\{2,3\}$, les agressions édulcorées grimpent à $70 \%$ et les actes les plus violents (plaquage, ceinturage, mêlées, etc.) chutent à $5 \%$.

Ce qui s'observe pour les deux équipes d'un point de vue qualitatif, se confirme pour chacune d'entre elles sur le plan quantitatif. La proportion de charges agressives 
délivrées est significativement plus importante lorsque la combinaison tactique (choix de ballons) désavantage l'une des deux équipes (cf. Figure 9). Dans le feu de l'action, la mission des joueurs favorisés étant si possible d'esquiver ; celle des joueurs défavorisés de les en empêcher. La durée d'une partie avoisinant 1 minute, il est remarquable d'y rencontrer en moyenne plus de 10 charges.

\begin{tabular}{|c|c|c|c|c|}
\hline $\begin{array}{r}\{\text { gain A, gain B\} } \\
\rightarrow\end{array}$ & $\{2,3\}$ & $\{3,1\}$ & $\{4,2\}$ & $\{1,4\}$ \\
\hline Équipe A & $\begin{array}{c}7,11 \\
\text { (256 des } 477 \text { charges } \\
\text { pour } 36 \text { parties) }\end{array}$ & $\begin{array}{c}4,44 \\
\text { (71 des } 199 \text { charges } \\
\text { pour } 16 \text { parties) }\end{array}$ & $\begin{array}{c}5,15 \\
\text { (67 des 178 charges } \\
\text { pour } 13 \text { parties) }\end{array}$ & $\begin{array}{c}8,93 \\
\text { (134 des 171 charges } \\
\text { pour 15 parties) }\end{array}$ \\
\hline Équipe B & $\begin{array}{c}6,14 \\
\text { (221 des } 477 \text { charges } \\
\text { pour 36 parties) }\end{array}$ & $\begin{array}{c}8 \\
\text { (128 des } 199 \text { charges } \\
\text { pour 16 parties) }\end{array}$ & $\begin{array}{c}8,54 \\
\text { (111 des 178 charges } \\
\text { pour 13 parties) }\end{array}$ & $\begin{array}{c}2,46 \\
\text { (37 des 171 charges } \\
\text { pour 15 parties) }\end{array}$ \\
\hline
\end{tabular}

FIGURE 9. Taux de charges agressives émises par partie selon la combinaison tactique (choix des ballons)

Quelle que soit l'équipe, l'émission des charges est d'autant plus importante que les gains potentiels sont défavorables. Les résultats sont significativement différents à $\mathrm{p}<0.01\left(\mathrm{KHI}^{2}\right)$.

Norbert Elias avait raison : l'inégalité des chances contribue activement aux manifestations d'agressivité motrice, tant dans les tactiques que dans les techniques de jeu.

\section{DISCUSSION. ÉGALITÉ DES CHANCES ET PACIFICATION DES MEURS}

Nous devons reconnaître que nous ne croyions pas un instant à la rationalité des équipes sur le terrain (coopération) et avions quelques craintes de débordements (par contre, nous avons été surpris de la logique des choix en secret de ballon). C'est la raison pour laquelle nous avons retenu des étudiants sportifs. Ils savent éviter les maladresses et tomber en souplesse. Au sortir de ce travail, nous n'avons d'ailleurs à déplorer aucun dommage corporel nécessitant un détour par l'infirmerie. Seuls quelques maillots arrachés ont émaillé le jeu. Mais les joueurs ont reconnu s'être bien amusés et le déroulement des séquences de jeu (choix secret du ballon, choix de la stratégie de terrain, exécution des actions motrices) est apparu beaucoup plus fluide que la complexité du système des scores ne le laissait présager.

Le système des scores exerce bien un pouvoir coercitif sur les conduites motrices des participants : lorsque la donne est faussée, les manifestations d'agressivité motrice vont bon train. D'autant plus qu'elles correspondent à une réorganisation pratique du rapport de force (en nombre d'attaquants et de défenseurs).

La généralisation du modèle d'affrontement sportif basé sur l'équilibre et l'impartialité permet traditionnellement aux joueurs d'incorporer un contrôle plus fort de leurs affects. On évite ainsi tous les débordements que nous avons pu observer dans notre jeu à travers les mêlées et agglutinations en tout genre.

Le sport réussit là où bon nombre de structures sociales ont échoué. L'inégalité perçue occasionne des règlements de compte dans la cité - voitures incendiées, altercations avec les forces de l'ordre, etc. Pourtant, c'est un fait que la vie quotidienne ne valorise pas autant que le sport la domination du plus fort et l'écrasement du plus faible. Ce n'est pas 
le moindre des paradoxes. Le sport met en avant la compétition et des modes de sélection que n'oserait même pas imaginer le plus sélectif des systèmes éducatifs. Mais perdre, échouer, se reconnaître inférieur semble plus digeste dès lors que les interactants sont soumis, dès le départ, à des règles équitables. "Plus digeste » signifie que la pilule de l'autocontrainte est plus facile à avaler; cela ne signifie pas nécessairement «plus agréable ».

Nous avons soumis le jeu vécu par les étudiants à leur appréciation, en leur proposant de répondre au différentiel sémantique d'Osgood [1975]. Il s'agit d'une technique d'analyse des significations affectives associées à certains stimuli inducteurs : ici, « sport » et « jeu » (sous-entendu : le jeu dont il est question dans cet article). Elle se présente sous forme d'un ensemble d'échelles bipolaires, dont chacune propose, entre deux qualificatifs opposés (plaisir/déplaisir, agréable/désagréable, positif/négatif, etc.), sept cases permettant aux joueurs de cocher le niveau correspondant à leurs appréciations. Les résultats sont très favorables au sport (moyenne de 4,4 sur 7, avec un écart-type de 1), ce qui n'est guère surprenant pour des étudiants sportifs. Mais ils le sont tout autant à l'égard du jeu où l'égalité des chances n'est pas respectée (moyenne de 4,7 sur 7, avec un écart-type de 0,8). L'agréable excitation procurée par la pratique des jeux sportifs n'est donc pas associée à l'équilibre des tensions générées par le système des scores. Et il se peut même qu'une trop grande mise en ordre affective finisse pas créer l'ennui.

\section{BIBLIOGRAPHIE}

BARBUT M., «Jeux et mathématiques. Jeux qui ne sont pas de pur hasard », Jeux et sports, Encyclopédie de la Pléiade, Paris, Gallimard, 1967, p. 836-864.

CONDORCET A., Mathématique et société, (1785), Paris, Hermann, 1974.

DELAHAYE J.-P., «Le jeu agité de la coopération », Jeux mathématiques et mathématiques des jeux, Paris, Belin, Pour la Science, 1998, p. 57-63.

ELIAS N., DUNNING E., Quest for excitement, Sport and leisure in the Civilizing Process, Basil Blackwell Ltd, 1986.

HALL E-T., The Hidden Dimension, New York, Doubleday \& Co, 1966.

KAGEL J., ROTH A., Handbook of Experimental Economics, Princeton, Princeton University Press, 1995.

LORENZ K., L'agression. Une histoire naturelle du mal, Paris, Flammarion, 1969.

OSGOOD C-E., The measurement of meaning, University of Illinois Press, 1975.

PARLEBAS P., Jeux, sports et sociétés. Lexique de praxéologie motrice, Paris, Institut National du Sport et de l'Education Physique, 1999.

PARLEBAS P., «Modélisation dans les jeux et les sports », Mathématiques et Sciences humaines 170, Paris, 2005, p. 11-45. 